\title{
Successful Tongue Replantation Following Segmental Autoamputation Using Supermicrosurgical Technique
}

\author{
Iris A. Seitz, MD, PhD ${ }^{1}$ Lucio A. Pavone, MD, FACS ${ }^{1}$ \\ ${ }^{1}$ Edward Plastic Surgery, Edward Elmhurst Health, Naperville, Illinois \\ 2 Plastic Surgeon, Morton Grove, Illinois
}

J Reconstr Microsurg Open 2017;2:e132-e135.

Tongue amputation is a rare event. In fact, only four successful tongue replants are reported in the literature. These include one incomplete amputation ${ }^{1}$ and three complete amputations. ${ }^{2-4}$ To our knowledge, replantation of a self-inflicted, segmental tongue amputation has not been reported. We report the successful replantation of a selfinflicted, segmental tongue amputation in an actively psy-
Loren S. Schechter, MD, FACS²

Address for correspondence Iris A. Seitz, MD, PhD, Edward Plastic Surgery, Edward Elmhurst Health, 120 Spalding drive, Suite 207, Naperville, IL 60540

(e-mail: iris.a.seitz@gmail.com; iseitz@edward.org).

chotic patient. Additionally, intra- and postoperative management strategies are reviewed.

\section{Patient and Methods}

A30-year-old native Polish male with new onset psychosis was brought to the Emergency Room after amputating his tongue
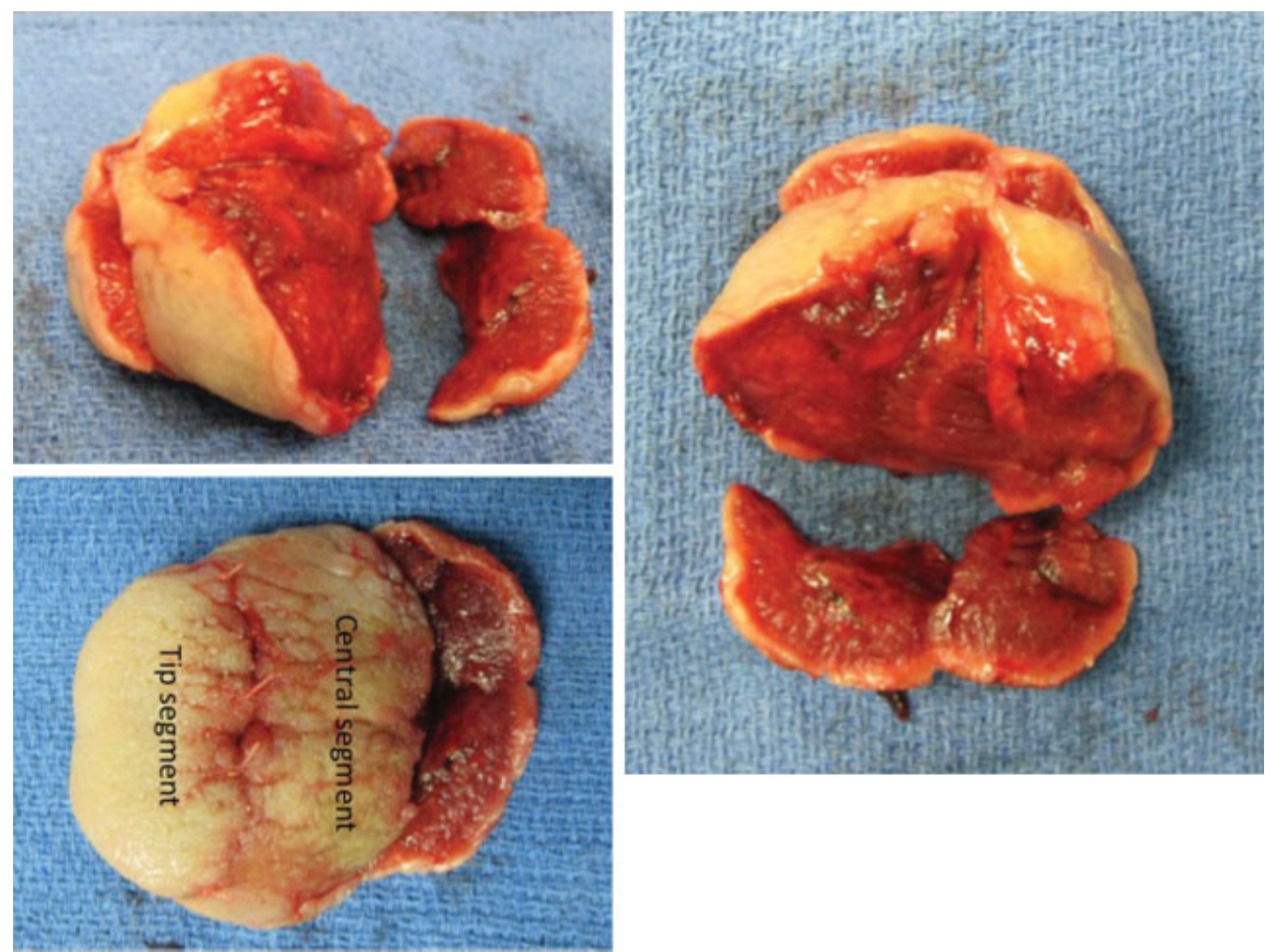

Fig. 1 Segmental tongue amputation.

received

December 30, 2016

accepted after revision

July 27, 2017
DOI https://doi.org/

10.1055/s-0037-1606584.

ISSN 2377-0813.
Copyright $\odot 2017$ by Thieme Medical Publishers, Inc., 333 Seventh Avenue, New York, NY 10001, USA. Tel: +1(212) 584-4662.
License terms

(c) $(1) \$$ 


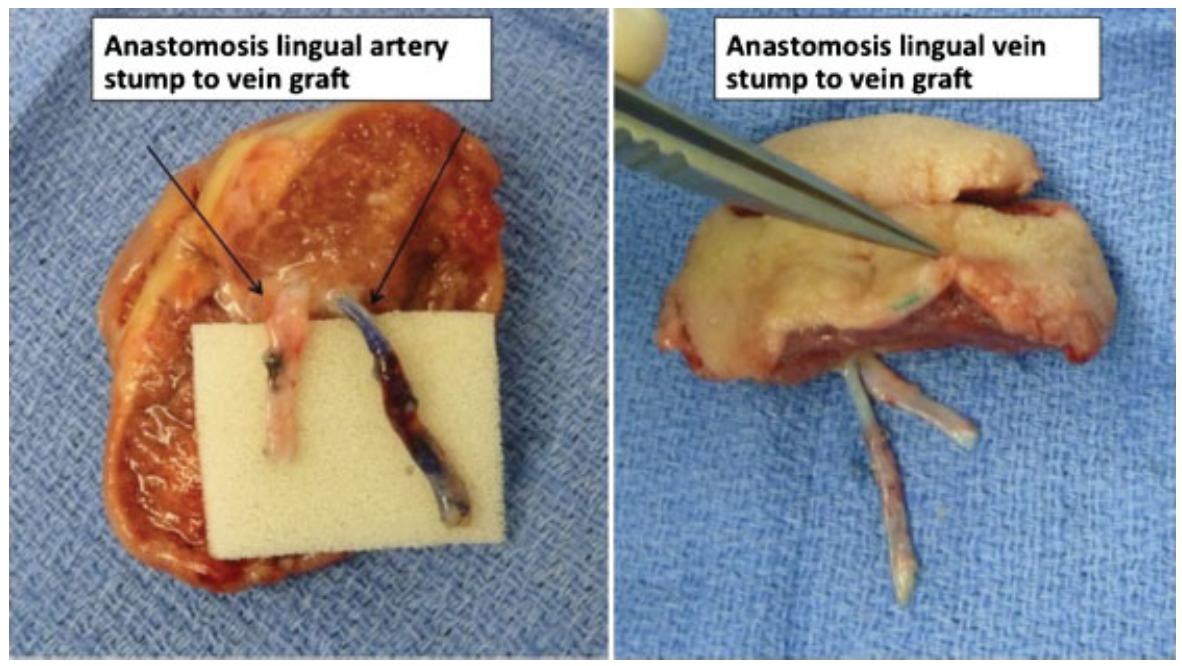

Fig. 2 Vein graft from dorsal hand to stump of lingual artery and vein within central amputated tongue segment.

with scissors. Three separate pieces were brought on ice, including the tip (L:1.35 x H:2.25 x W:3.75 cm), a large central segment (L:2.7 $\times \mathrm{H}: 3.8 \times \mathrm{W}: 5.9 \mathrm{~cm})$, and a thin slice from the base of the tongue (L:0.5 x H:1 x W:5 cm)(-Fig. 1). The patient underwent urgent intubation in the Emergency Department. The lingual arteries were clamped by the on-call otolaryngologist and a plastic surgery consultation was requested.

Following discussion with the patient's parents, a decision was made to proceed with replantation. The patient was transported to the operating room and underwent a tracheostomy. Concurrently, the amputated tongue segments were evaluated under the operating microscope. The large central segment was found suitable for replantation. The tip segment was reattached to the replanted segment as a composite graft ( - Fig. 2) and the thin slice of amputated tongue was discarded. The central segment was flushed with heparinized saline $(500 \mathrm{u} / \mathrm{cc}$ ) through the arterial stump of the left lingual artery. This allowed visualization of the draining lingual vein within the amputated tongue segment. A second plastic surgeon harvested an interposition vein

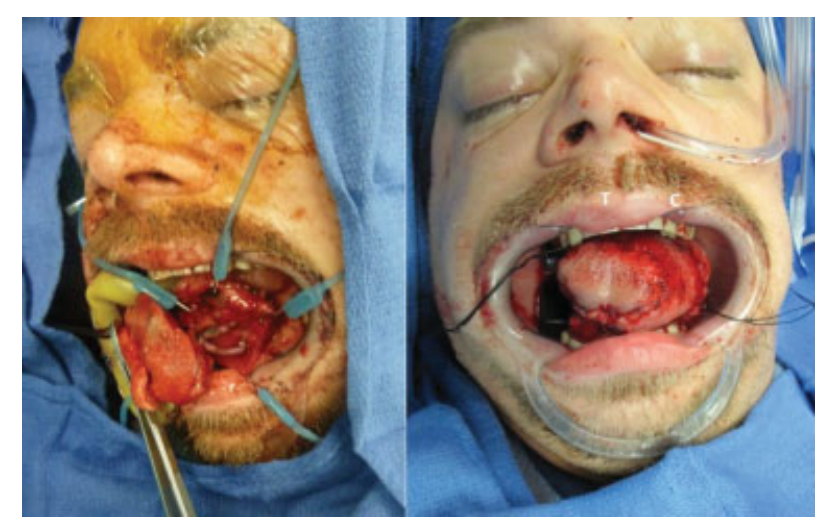

Fig. 3 Arterial and venous anastomosis of central tongue segment with dorsal hand vein graft to left lingual artery and vein stump, tip segment as composite graft. graft from the dorsum of the left hand. This was subsequently utilized for both the arterial and venous anastomoses (performed to the left recipient lingual vessels located at the base of the remaining tongue).

The arterial diameter in the amputated central segment was $0.7 \mathrm{~mm}$, and the venous diameter was $0.5 \mathrm{~mm}$. The operative sequence included anastomosis of the vein grafts to the amputated tongue vessel stumps using interrupted 11-0 nylon sutures ( $\mathbf{- F i g .} \mathbf{2}$ ). This was performed using a supermicrosurgical parachute suture technique. The vein grafts were then anastomosed to the recipient left lingual artery and vein at the base of the tongue using interrupted 10-0 nylon sutures ( $\mathbf{F}$ Fig. $\mathbf{3}$ ). Total ischemia time was 7 hours.

Postoperative management included a bite block, intravenous dextran drip, and leech therapy. The patient also received multiple blood transfusions. The patient was sedated and paralyzed in the initial postoperative period and antipsychotic medications were prescribed by the consulting psychiatry service. A small wound dehiscence on the non-revascularized right side healed by secondary intention. The patient received inpatient speech therapy and close psychiatric monitoring. He was discharged after 4 weeks.

\section{Results}

At 6 months post-replantation, the patient had excellent tongue mobility, was tolerating a regular diet, and had normal, intelligible speech as assessed by a certified speech therapist (-Fig. 4). He is now 4 years post-replantation and has regained near-complete sensation and taste.

\section{Discussion}

Tongue amputation is rare, and replantation is a technical challenge. However, because the tongue plays a critical role in swallowing, speech, taste, and airway protection, replantation is the best way to reestablish oral function. The 


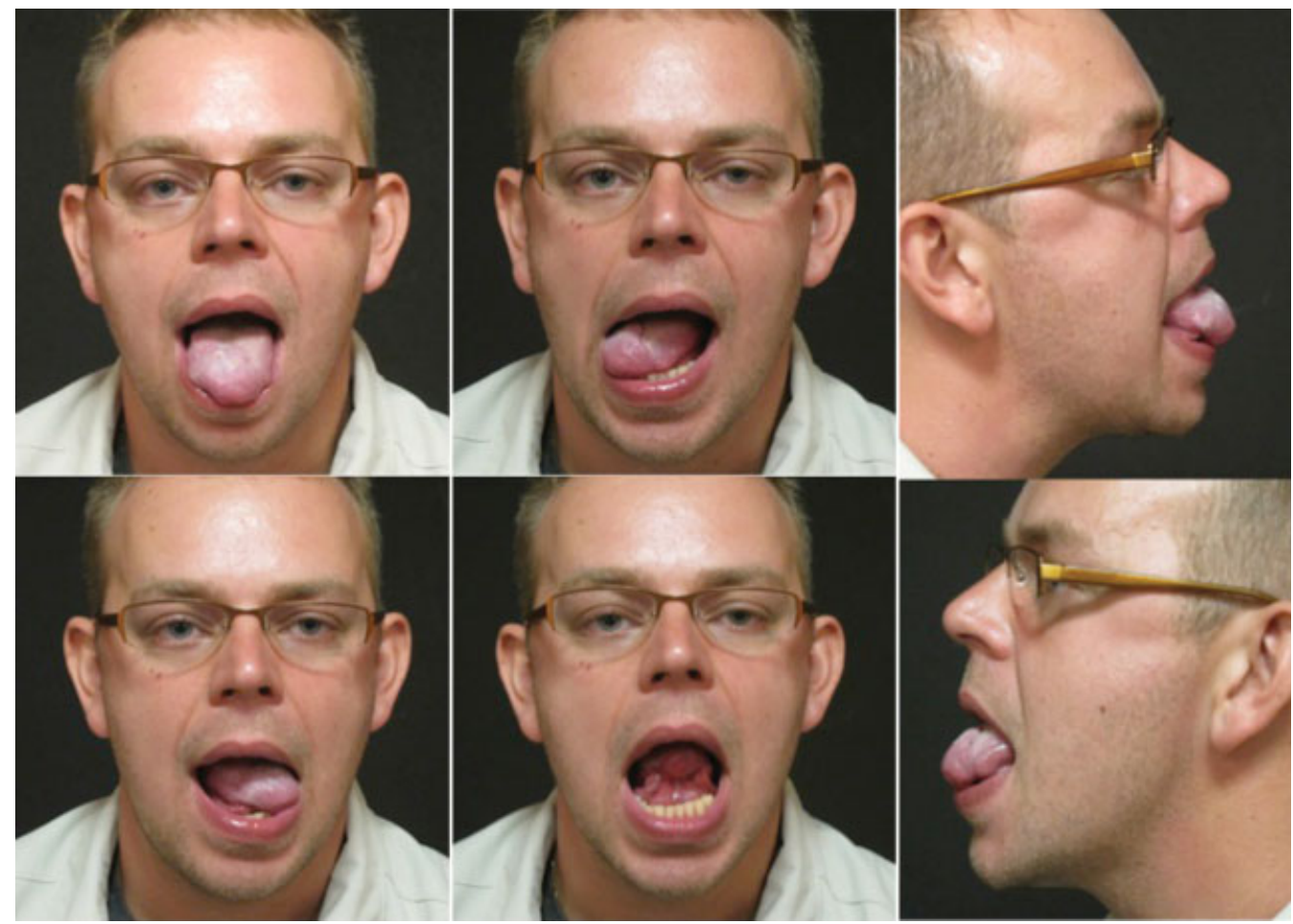

Fig. 4 One year post-replantation.

previous case reports vary in regard to the mechanism of injury, size of the amputated tongue, technical details, ischemia time, use of leeches and vein grafts, anticoagulative therapy, and outcome. In 1998, Buntic and Bunke reported the first successful tongue replant of an amputated segment measuring $5 \times 4 \mathrm{~cm}$ with a $1.5 \mathrm{~mm}$ vessel diameter. A direct anastomosis was performed between the lingual arteries, and a vein graft to the left facial vein was performed. Ischemia time measured 8 hours and postoperative aspirin and dextran therapy was utilized. ${ }^{2}$ Davis and Armstrong reported a failed tongue replantation measuring $5 \times 3.5 \mathrm{~cm}$ with a $0.5 \mathrm{~mm}$ vessel diameter. In this case, direct anastomoses of the left lingual artery and right lingual vein were performed. Ischemia time measured 10 hours and postoperative dextran and aspirin therapy was utilized. Necrosis of the replant was noted on the fifth postoperative day. ${ }^{5}$ Egozi et al reported replantation of an incomplete tongue amputation related to a motorcycle injury. Vessel diameter was less than $1 \mathrm{~mm}$, and the left lingual artery and right lingual vein were used for the anastomoses. Ischemia time was 16 hours and postoperative dextran and aspirin therapy were utilized. One $\mathrm{cm}$ necrosis of the tip was noted. ${ }^{1}$ Kim et al replanted a small tongue segment measuring $2.5 \times 1.7 \mathrm{~cm}$, with vessels measuring 0.8 and $0.7 \mathrm{~mm}$ diameter. Only the left lingual artery was anastomosed and no venous anastomosis was performed. Ischemia time was 9 hours. Leech therapy, PGE1, dipyramidol, and aspirin therapy were utilized in the postoperative period. ${ }^{3}$ Finally, Hong and Eun replanted a tongue segment measuring $3.5 \times 2 \mathrm{~cm}$ with vessel diameters of 0.8 and $0.9 \mathrm{~mm}$. The anastomoses were performed to the left lingual artery and vein, with 14 hours of ischemia time. Postoperative paralysis and antipsychotic therapy were initiated. ${ }^{4}$

This is the first case to describe replantation of a segmental amputation of the tongue. In replantation surgery, segmental injuries can be a contraindication for replantation. While this may be the case for digits or extremities, replantation of a segmental tongue amputation may be worthwhile, even though the patient's acute psychosis presented an additional management challenge.

\section{Conclusion}

We believe that successful replantation is based on multiple factors. This includes a) the timely evaluation and reperfusion of the amputated part typically in a two-team approach. One team prepares the recipient vessels and possible vein graft, and the second team works under the microscope preparing the amputated part. This entails maintaining the amputated part on ice, flushing the amputating part with a heparinized solution, and performing the anastomoses between the amputated part and the vein grafts, b) meticulous micro- or supermicrosurgical technique, c) postoperative leech therapy, d) sedation and paralysis during the immediate postoperative period, and e) early antipsychotic pharmacological treatment in this particular case. Furthermore, as previously reported, and confirmed in this case, the tongue can survive on unilateral revascularization. However, contralateral wound dehiscence may be more likely. ${ }^{3}$ 


\section{Conflict of Interest}

None.

\section{References}

1 Egozi E, Faulkner B, Lin KY. Successful revascularization following near-complete amputation of the tongue. Ann Plast Surg 2006;56 (02):190-193
2 Buntic RF, Buncke HJ. Successful replantation of an amputated tongue. Plast Reconstr Surg 1998;101(06):1604-1607

3 Kim JS, Choi TH, Kim NG, et al. The replantation of an amputated tongue by supermicrosurgery. J Plast Reconstr Aesthet Surg 2007; 60(10):1152-1155

4 Hong JM, Eun SC. Self-mutilation of the tongue in a patient with schizophrenia. J Craniofac Surg 2014;25(02):e116-e118

5 Davis C, Armstrong J. Replantation of an amputated tongue. Plast Reconstr Surg 2001;108(05):1441-1442 